\title{
El Federalismo bajo revisión: preocupaciones, desafíos y propuestas desde la Facultad de Derecho de la Universidad de Buenos Aires (1890-1916)
}

\section{Federalism under review: concerns, challenges and proposals at the University of Buenos Aires School of Law (1890-1916)}

\author{
Maria Pollitzer \\ Universidad Nacional de San Martín \\ Escuela de Política y Gobierno \\ Centro de Estudios de Historia Política \\ Buenos Aires, Argentina \\ maria_pollitzer@hotmail.com
}

\begin{abstract}
Resumen
El artículo busca reponer las principales discusiones acerca del federalismo argentino libradas al interior de la Facultad de Derecho y Ciencias Sociales de la Universidad de Buenos Aires. Se concentra en aquellos aspectos de esta temática que atrajeron la atención de cerca de cincuenta alumnos quienes, entre 1890 y 1916, dedicaron sus tesis doctorales al análisis de la misma. Se identifican las claves analíticas en torno a las cuales ordenaron sus discursos, los pilares sobre lo que construyeron sus diagnósticos y las propuestas de reforma alentadas. Además de estas tesis, el trabajo explora los discursos académicos pronunciados en este período, algunos de los manuales utilizados en las cátedras de derecho
\end{abstract}

Cómo citar este artículo/ How to cite this article: Pollitzer, M. (2021). El Federalismo bajo revisión: preocupaciones, desafíos y propuestas analizadas en la Facultad de Derecho de la Universidad de Buenos Aires (1890-1916). Revista de Historia Americana y Argentina, 56 (1), pp. 271-302. https://doi.org/10.48162/rev.44.008 
constitucional y las revistas de estrecha vinculación con la vida universitaria. El análisis de este rico corpus documental resulta una vía poco transitada para dar cuenta de la circulación de ideas y discursos en el ámbito porteño hacia el cambio de siglo.

Palabras clave: federalismo; unitarismo; gobierno representativo; Facultad de Derecho; tesis doctorales.

\begin{abstract}
The article addresses the main discussions concerning Argentine federalism held within the Faculty of Law and Social Sciences of the University of Buenos Aires. It focuses on those aspects of this subject that attracted the attention of about 50 students who chose to dedicate their doctoral theses to its analysis between 1890 and 1916. Analytical keys around which speeches were organized, pillars on which diagnoses were built and proposals for reform encouraged are identified. In addition to these theses, the article explores academic speeches delivered in this period, some of the manuals used in the chairs of constitutional law and journals closely linked to university life. The analysis of this rich documentary corpus is a fruitful way to account for the circulation of ideas and discourses in the Buenos Aires area towards the turn of the century.
\end{abstract}

Key words: Federalism; Unitarism; Representative Government, School of Law; Doctoral Dissertation.

Recibido: 06/08/2020. Aceptado: 15/03/2021

\title{
Un asunto de interés y palpitante actualidad
}

A fines del siglo XIX y comienzos del XX, los discursos pronunciados por los decanos de la Facultad de Derecho y Ciencias Sociales de la Universidad de Buenos Aires con ocasión de la apertura de los cursos, o aquellos pronunciados por docentes y graduados en los actos de colación de grados, reafirman como tarea principal e irrenunciable de este espacio el formar ciudadanos y estadistas capaces de "servir a la República estudiándola"1. La sociedad esperaba de los nuevos doctores en jurisprudencia "una alta

${ }^{1}$ Garcia, J. A. (1898). Discurso de colación de grados. Anales de la Universidad, XIII, p.188. 
contribución en ideas y esfuerzos" 2 que permitiera hacer frente a los grandes desafíos que la nación tenía por delante. En paralelo, Antonio Dellepiane (profesor de Filosofía del Derecho) recordaba a los alumnos que "es deber de nuestra generación y la vuestra trabajar por la verdad de las instituciones, conseguir que ellas dejen de ser derecho escrito para convertirse en derecho vivido" 3 .

Varios fueron los temas que, en materia de derecho constitucional, ocuparon el centro de las reflexiones de estos claustros docentes y estudiantiles entre 1890 y 1916 . Entre ellos, podemos señalar: el sujeto de imputación de la ciudadanía; la naturalización de los extranjeros; las vías de participación ciudadana; el problema del abstencionismo electoral; la legislación electoral vigente y la necesidad de su reforma; la cuestión del sufragio, su naturaleza y extensión; las atribuciones del poder ejecutivo nacional y la posibilidad de instaurar un régimen parlamentario; la organización de la Justicia; la relación entre el estado y la Iglesia, o bien, la distancia observada entre la aspiración constitucional tendiente a de consolidar un régimen federal y los resultados concretos que la experiencia de las últimas décadas había arrojado. Es sobre este último aspecto que nos interesa concentrar la atención en esta oportunidad.

En efecto, entre 1894 y 1916 se defendieron alrededor de cincuenta tesis doctorales en las que los alumnos analizaron la forma del estado argentino, los orígenes y evolución del federalismo, los poderes del estado federal, la teoría y práctica de las intervenciones provinciales y la conveniencia o no de modificar la constitución en vistas de instaurar un régimen unitario ${ }^{4}$. Todos ellos, "temas de interés y palpitante actualidad". Así lo consignan, entre otros, Enrique Prack, Carlos Octavio Bunge o Carlos María Vico, doctorandos que más tarde se incorporarían al claustro docente de la Facultad $^{5}$. Sus textos suelen iniciarse con afirmaciones como las siguientes:

2Díaz Arana, J. J. (1916). Discurso de colación de grados. Revista del Centro de Estudiantes de Derecho, 61, p. 270.

${ }^{3}$ Dellepiane, A. (1909). Discurso de colación de grados. Revista Jurídica y de Ciencias Sociales, XXVI, (2), p. 120.

${ }^{4}$ En su obra Del Régimen Federativo al Unitario, Rodolfo Rivarola (1908) afirma que algunos de estos trabajos contienen "mérito positivo", aunque se excusa de referirse en detalle a ellos por una cuestión de espacio y porque prefiere detenerse en "las opiniones que sobre la base de la erudición agregan la de la experiencia" (p. 220).

${ }^{5}$ El primero, en la cátedra de derecho penal; Carlos Octavio Bunge, en Introducción al Derecho y Carlos María Vico, en derecho internacional privado. 
"Habiendo oído por doquier: "marchamos al unitarismo»; "con el sistema unitario estaríamos mejor gobernados» o frases semejantes" 6 ; "Es frecuente oír manifestarse, aun en personas ilustradas, la opinión de lo conveniente que hubiese sido la implantación del régimen unitario en nuestro sistema político" (Bunge, 1897, p. 3), o acaso:

Hace ya tiempo se nota en cierto número de intelectuales la predilección por el régimen unitario para nuestros días, y este año se ha presentado esa predilección entre los graduados de Derecho, bien en forma de proposiciones accesorias, bien en tesis ${ }^{7}$.

Acevedo insiste, por su parte, en que "desde un tiempo a esta parte viene agitándose entre nosotros el problema de nuestro régimen político" y da cuenta de la "atmósfera de descrédito que alrededor de nuestro federalismo viene haciéndose"8.

En consonancia con los debates que pudieran tener lugar en el ámbito parlamentario, en la prensa periódica ${ }^{9} 0$ en los mitines políticos, los docentes y autoridades de la Facultad alentaron el estudio de estas temáticas. Para ello, propusieron como tópico para el premio otorgado por el Centro Jurídico en 1896 "El deslinde de las facultades nacionales y provinciales"10 y en el listado de temas optativos para que los alumnos preparan sus disertaciones incluyeron títulos como "El concepto del

${ }^{6}$ Prack, E. (1893). Sistema federal. Revista Jurídica y de Ciencias Sociales, IX (3), p. 209.

${ }^{7}$ Vico, C. M. (1905). Una tesis unitaria. Revista Jurídica y de Ciencias Sociales, XXII, (1), p. 197.

${ }^{8}$ Acevedo, M. (1913). Sistema federal en la República Argentina. Revista del Centro de Estudiantes de la Facultad de Derecho y Ciencia Sociales, 40, p. 136.

${ }^{9}$ Tan sólo a título ilustrativo, en el segundo año de la publicación de la Revista de Derecho, Historia y Letras, su director y, por entonces, profesor de derecho internacional privado, Estanislao Zeballos (1898) se pregunta si ha llegado la oportunidad de organizar un partido unitario y señala que "la Revista ha planteado la cuestión consultando a numerosos argentinos descollantes" (p. 224). Otro ejemplo lo provee la Revista Argentina de Ciencias Políticas, cuyo director, Rodolfo Rivarola, propició una encuesta en 1911 en la que indagaba, entre otros puntos, acerca del régimen constitucional vigente, si éste debía ser federal, nacional o unitario. Cfr. al respecto, Alonso (2006).

${ }^{10}$ Rómulo Naón (18906), quien se desempeñaría en los años siguientes como profesor de derecho constitucional en esta misma casa de estudios, fue quien obtuvo dicho premio. 
federalismo entre los principales sociólogos de nuestros días" (para 19091911) ${ }^{11}$, "El derecho federal: El recurso extraordinario autorizado en los artículos 14 de la ley número 48 y 6 de la ley 4055" (para 1915) o bien, "Explicación y comentario del artículo 104 de la constitución federal" (para 1916). A su vez, los estudiantes y profesores expresaron sus opiniones sobre estos asuntos en artículos publicados en la Revista del Centro de Estudiantes, en la Revista de la Universidad, los Anales de la Facultad y en publicaciones periódicas de estrecha vinculación con la vida académica de esta Facultad, como la Revista Jurídica y de Ciencias Sociales, la Revista de Derecho, Historia y Letras o la Revista Argentina de Ciencias Políticas. Ocasionalmente, también se refirieron a ellos en los discursos pronunciados en los actos de colación de grados.

Nos interesa preguntarnos, a continuación, de qué manera fue abordado este estudio (principalmente, por parte de los doctorandos), bajo qué claves analíticas ordenaron sus discursos y sobre qué pilares construyeron sus diagnósticos. Asimismo, detectar cuáles fueron las modificaciones que alentaron y cómo fueron recibidas y discutidas las lecciones y propuestas presentadas por algunos profesores de la casa (que a su vez desempeñaron diversas funciones públicas) como José Manuel Estrada, Aristóbulo Del Valle, Manuel Augusto Montes de Oca, Carlos Rodríguez Larreta o Rodolfo Rivarola.

Cabe recordar brevemente que, hasta 1909, la carrera de abogacía duraba cinco años y los títulos de doctor y abogado se expedían simultáneamente una vez que el alumno defendía ante un tribunal su trabajo de tesis. A comienzos del siglo $\mathrm{XX}$, el número de inscriptos se había elevado considerablemente $y$, si en 1890 la Facultad de Derecho contaba con apenas 310 alumnos, en 1909 éste rondaba en torno a los 1050. A partir de entonces, con la aprobación de un nuevo plan de estudios, el doctorado quedó separado de la carrera de grado (a la que se sumaba un año) y se crearon asignaturas específicas para quienes, extendiendo sus estudios por un año más, aspirasen al título doctoral12. También se modificó el mecanismo para la elección de los temas sobre los que deberían versar las tesis doctorales. Cada año, los profesores titulares debían sugerir tres puntos del programa de sus respectivas materias y elevarlos a

\footnotetext{
${ }^{11}$ Ningún doctorando eligió este tema para su disertación.

${ }^{12}$ En 1913 se aprobó un nuevo plan de estudios que volvía la carrera de abogacía a 5 años y organizaba el doctorado en 2 años. Entró en vigor en 1918)
} 
consideración del Consejo Directivo de la Facultad, órgano encargado de seleccionar uno solo por asignatura ${ }^{13}$. El listado final de temas oscilaba entre 35 y 45 "cuestiones nacionales o de interés para la vida jurídica o social de la nación" 14 . Se esperaba que dichos trabajos fueran el fruto de una investigación personal del autor, desarrollada- al igual que en las lecciones impartidas en los distintos cursos- de manera "serena, ilustrada y desapasionada" 15 y en la que se reflejara "un estudio crítico de nuestras leyes y jurisprudencia, nuestros antecedentes y peculiaridades del país, examen de las doctrinas y legislación comparada" (Candioti, 1920, p. 281).

Respecto de las tesis que se ocuparon de las distintas modulaciones que presentaba la cuestión federal, observamos que en, la gran mayoría, la discusión doctrinaria sobre las bondades atribuibles al régimen federal o al unitario ocupa un lugar acotado o acaso inexistente. No discurre por esta vía el grueso de los análisis ni los principales disensos. De hecho, ninguno de los tesistas consultados parece discutir la superioridad teórica del sistema federal. Incluso aquellos que se manifiestan favorables a una reforma constitucional que establezca entre nosotros un régimen unitario aclaran que no han querido confrontar unitarismo y federalismo en el plano de la teoría y admiten que

(...) en el terreno doctrinario, el federalismo es preferible, porque se aproxima más al desgobierno que será el desiderátum de la Humanidad cuando lleguemos a convencernos de que es posible ejercer por la educación el control que nos obliga a respetar los preceptos fundamentales del derecho y de la convivencia social (Padilla, 1900, p. 112).

En la misma línea se expresa Rafael Candioti (1898), quien se reconoce "apasionadísimo del federalismo teórico" (p. 137) y "familiarizado" (p. 44) con las obras de Joseph Story, Joel Tiffany o George Paschal.

\footnotetext{
${ }^{13}$ Se contemplaba la posibilidad de que un alumno pudiera optar por un tema no incluido en el listado final, siempre y cuando contara con la autorización y aprobación del Consejo.

${ }^{14}$ Bidau, E. (1912). Discurso de apertura de los cursos de 1912. Anales de la Facultad de Derecho y Ciencias Sociales, II (2), p. 623.

${ }^{15}$ Bidau, E. (1911). Discurso de apertura de los cursos de 1911. Anales de la Facultad de Derecho y Ciencias Sociales, II, (1), p. 586.
} 
Entre las escasas referencias que aparecen al respecto se señala, por ejemplo, que el sistema federal hace posible la conciliación de dos elementos aparentemente antagónicos: la libertad popular y la fuerza y eficacia del poder central. "La descentralización- advierte Octavio Navarro (1897)- permite que el pueblo tenga en el manejo y la dirección de la cosa pública una participación suficientemente eficaz para imponer a los gobernantes la obligación de proceder conforme a la voluntad nacional" ( $p$. 25). En un artículo publicado en 1913 por la Revista del Centro de Estudiantes, Martín Acevedo (quien defendería su tesis sobre el voto obligatorio al año siguiente) cuestiona las ideas de R. Rivarola recuperando las ventajas que, según James Bryce, aseguraba el régimen federal. Así reproduce textualmente las primeras 5 del listado original presente en The American Commonwealth (1888). Un régimen federal:

1) Permite unir varias entidades políticas en una sola nación, sin destruir su administración, su legislación, su patriotismo local. 2) Es el mejor medio para dar a un país vasto y nuevo su desenvolvimiento normal. Permite atender mejor a las necesidades de cada región. 3) Previene el nacimiento de un gobierno central despótico 4) El gobierno autonómico es un estimulante del interés popular en los negocios regionales y un sostén de la vida política rural, enseña a los ciudadanos que sus deberes cívicos, la vigilancia perpetua y el sacrificio de su tiempo son el precio de la libertad individual y la prosperidad colectiva. 5) Asegura una buena administración de los negocios locales al darle a los habitantes de cada localidad los medios para controlar la conducción de sus asuntos ${ }^{16}$.

Y si bien hay quien asocia unitarismo a despotismo, con el argumento de que la concentración del poder que éste supone robustece la acción del gobierno, mientras que la descentralización alentada por el federalismo conduce a la libertad (Silgueira, 1894, p. 24), son mayoría los que advierten que la democracia es igualmente posible bajo uno u otro sistema (Leiva,

\footnotetext{
${ }^{16}$ Acevedo, M. (1913). Sistema federal en la República Argentina. Revista del Centro de Estudiantes de la Facultad de Derecho y Ciencia Sociales, 40, p. 139. Las tres ventajas restantes, no reproducidas por Acevedo, pero sí aludidas en la tesis de Octavio Navarro (1897, p. 25) son: 1: El federalismo permite al pueblo experimentar, en materia de legislación y administración, lo que no podría hacerse con seguridad en un país fuertemente centralizado. 2. El federalismo, si bien disminuye la fuerza colectiva de la nación también disminuye el riesgo al que su tamaño y la diversidad de sus partes lo expone. 3. Al crear muchas legislaturas locales alivia a la legislatura nacional de una gran cantidad de funciones que le resultarían muy pesada.
} 
1905, p. 41) y que la libertad no es incompatible con la concentración del poder (Padilla, 1900, p. 106). De todos modos, como dijimos, los análisis presentados por los tesistas no focalizan sobre esta dimensión ${ }^{17}$. Su objeto de estudio es el escenario local y su atención se dirige, en términos relativamente equilibrados, tanto al pasado colonial y las experiencias vividas tras la Revolución de Mayo, como hacia la configuración presente y sus desafíos.

\section{La ineludible mirada retrospectiva}

Sobrevuela en todos los trabajos un acuerdo general respecto de la imposibilidad de comprender adecuadamente la constitución por entonces vigente o las anteriores, explicar sus preceptos o valorar las instituciones que nos rigen sin conocer la propia historia nacional, los antecedentes, la raíz o punto de partida. Lucio V. López y Aristóbulo del Valle habían puesto especial énfasis sobre este punto mientras estuvieron al frente de la cátedra de derecho constitucional, 1884-1894 el primero, y 1895-1896, el segundo. (Leiva, 1989; Tanzi, 2011; Tau Anzoátegui, 1996).

El primer aspecto sobre el que pocos eluden pronunciarse remite al origen de nuestro federalismo. Tan sólo unos pocos ofrecen un análisis más exhaustivo, en el que distinguen entre un origen remoto y otro más próximo y calibran la importancia relativa que cada elemento pudo haber desempeñado. Otros tan solo señalan aquél que consideran más relevante. En un artículo publicado por los Anales de la Facultad en $1902^{18}$, C. Rodríguez Larreta (profesor suplente de la cátedra de derecho constitucional desde 1896 y titular entre 1899 y 1908) reconoce que se trata de un asunto sobre el que aún no se había arribado a un consenso, discute las lecturas que hasta el momento habían ofrecido Francisco Ramos Mejía (El Federalismo Argentino), Bartolomé Mitre (Historia de Belgrano) y Vicente Fidel López (Historia Argentina) y propone su propia interpretación. No acuerda con Ramos Mejía en presentar al federalismo argentino como el producto de una herencia en la que el componente racial del pueblo conquistador, su carácter y costumbres resultaban fundamentales. Las

\footnotetext{
${ }^{17}$ Lo mismo ocurre en las Lecciones de Derecho Constitucional impartidas por M. Montes de Oca, profesor a cargo de Derecho Constitucional entre 1896 y 1912. ${ }^{18}$ Rodríguez Larreta, C. (1902). Origen del federalismo argentino. Anales de la Facultad de Derecho y Ciencias Sociales, 1, pp. 389-405.
} 
opiniones de los tesistas aparecen divididas sobre este punto ${ }^{19}$ y lo mismo ocurre a la hora de valorar el legado de los cabildos coloniales. Amparado por las lecciones de Alberdi, Estrada y Montes de Oca, C. O. Bunge insiste en su tesis en presentar a estas instituciones como la cuna de la representación y el federalismo. En contrapartida, Rafael Candioti, Uladislao Padilla, José Abraham Leiva y Octavio Amadeo se encolumnan detrás de Del Valle, García y Rodríguez Larreta y se distancian de lo que consideran "una fábula desvanecida" 20 una "bella mentira poética" (Amadeo, 1900, p. 20).

No creemos que los cabildos sean el origen de nuestra vida representativa y democrática (...) No creemos tampoco que hayan servido para difundir entre los pueblos de América el amor por las instituciones republicanas (...) La venalidad en la distribución de los cargos y las restricciones impuestas a la elección de sus miembros es el más elocuente desmentido que pueda arrojarse a los que sostienen que su influencia ha sido decisiva en la constitución del gobierno americano- sentencia J. A. Leiva (1905, p.16).

No es posible trazar una genealogía directa y exclusiva que enlace nuestro federalismo con la historia, el carácter o las instituciones de la madre patria, insiste Rodríguez Larreta. En la misma línea se expresa Emilio Matienzo, hermano de José Nicolás, quien mereció un diploma de honor al graduarse en 1894. En su tesis afirma que el unitarismo y el federalismo habían llegado juntos a América:

España transportó a América las dos ideas que la gobernaban. El unitarismo lo simbolizó el Virrey y el federalismo en los cabildos. El unitarismo no encontró tierra hospitalaria en la americana: de la selva, del llano y de la montaña surgieron miles de leguas que le hicieron cruda e incesante guerra. Los presidentes se independizaron de los virreyes y los gobernadores e intendentes, de los presidentes. El federalismo no fue tan desgraciado: la América y sus grandezas le

\footnotetext{
${ }^{19} \mathrm{~A}$ modo de ejemplo, los trabajos de H. Silgueira $(1894$, p. 24) y C. O. Bunge (1897, p. 258) suscriben la tesis de Ramos Mejía (Bunge se ampara también en la opinión de Estrada y Montes de Oca), mientras que los de C. Melo (1897, p. 25, 33) y J. A. Leiva (1905, p. 14) la cuestionan. Este último había elegido a Carlos Rodríguez Larreta como su padrino de tesis y en su trabajo cita varios párrafos del artículo publicado por su maestro en los Anales de la Facultad.

${ }^{20}$ Rodríguez Larreta, C. (1902). Origen del federalismo argentino. Anales de la Facultad de Derecho y Ciencias Sociales, 1, p. 391.
} 
recibieron batiendo palmas, el desierto le entonó himnos y levantó en su honor baluartes y murallas infranqueables. (Matienzo, 1894, p.18).

Ello nos remite a otro factor en el que varios tesistas anudan el origen del federalismo argentino: la configuración espacial de nuestro suelo, su particular fisonomía, su gran extensión, aquella sobre la cual se impuso la "ciudad- fortaleza" o la "ciudad-cuartel" que se mantuvo aislada y "separada por el desierto y el salvaje"21. He ahí el origen más remoto de la federación argentina según Rodríguez Larreta. "Las instituciones coloniales eran absolutas y unitarias pero la fuerza que garantizaba su ejercicio se veía debilitada por los efectos del aislamiento y la distancia"22.

En cuanto a los orígenes más próximos y más estrechamente vinculados a nuestra historia patria, tres momentos se disputan la primacía. Mitre creía reconocer la aparición del federalismo con el desprendimiento de Paraguay y con las decisiones adoptadas por la Junta de Mayo (relativas a la incorporación de los diputados del interior y a la creación de las juntas provinciales). De acuerdo con Del Valle, Rodríguez Larreta desestima esta interpretación $\mathrm{y}$, tras recordar los propósitos de la Junta Provisional expresados en el decreto por medio del cual se creaban las juntas provinciales, aclara que éste alude a las relaciones entre el gobierno y los súbditos "pero no a la coexistencia de diversas autonomías bajo la ley común de la nacionalidad, que es lo que constituye (...) el régimen federal"23. Entre los tesistas, C. O. Bunge (1897) es el único que se manifiesta explícitamente a favor de la explicación mitrista, que encuentra respaldada por Estrada, Lucio V. López y Montes de Oca.

Por su parte, Vicente Fidel López y del Valle señalan un segundo momento como acta de nacimiento de nuestro federalismo: la sublevación de los caudillos del litoral contra el gobierno establecido en Buenos Aires desde 1820. Juan Cruz Cruz, graduado en 1896 y posteriormente docente de derecho comercial en la misma Facultad, participa de esta opinión y encuentra en la vida nómade de los caudillos el antecedente "no de un

\footnotetext{
${ }^{21}$ Rodríguez Larreta, C. (1902). Origen del federalismo argentino. Anales de la Facultad de Derecho y Ciencias Sociales, 1, pp. 396.

${ }^{22}$ Rodríguez Larreta, C. (1902). Origen del federalismo argentino. Anales de la Facultad de Derecho y Ciencias Sociales, 1, pp. 403.

${ }^{23}$ Rodríguez Larreta, C. (1902). Origen del federalismo argentino. Anales de la Facultad de Derecho y Ciencias Sociales, 1, pp. 394.
} 
federalismo científico, sino más bien de una tendencia que acusa uno de sus caracteres principales: la descentralización del poder y la creación de autonomías circunscriptas por el radio de acción de cada caudillo" (Cruz, 1896, p. 97-8). O. Amadeo (1900) lo afirma abiertamente: las autonomías provinciales tienen una "paternidad inconfesable: el caudillaje" (p. 24). No respondieron a las exigencias de pueblos habituados al self-government, ni surcados por divergencias de raza, cultura, religión, idioma, instituciones, sino que son el fruto "puro y exclusivo de una situación anormal: el alzamiento del gaucho malo contra el hombre culto de las ciudades" (p. 24). U. Padilla (1900) y Eliseo Marenco Aberastain (1905) abonan esta hipótesis. Sostienen que los caudillos, lejos de perseguir la autonomía provincial, buscaron ante todo extender su poder y explican que

(...) uno de los hechos que demuestra que los pueblos de las provincias no tenían tendencias al federalismo y sí al unitarismo es que las provincias donde no surgieron al principio de nuestra vida independiente caudillos gauchi-políticos, se mantuvieron fieles a los gobiernos centrales mientras ellos no desaparecieron (Marenco Aberastain 1905, p.42).

Para Rodríguez Larreta, la explicación que sólo apunta a la voluntad caprichosa de los caudillos no advierte que

$(,,$,$) los hechos sociales, cuando llegan a imprimir en un pueblo$ modalidades tan extrañas y tan fuertes, no son jamás el fruto efímero de una resolución individual: tienen raíces profundas, son el resultado de necesidades imperiosas contra las cuales nada puede la deliberación aislada de los hombres ${ }^{24}$.

Sí admite, no obstante, que aquí yace la primera explosión del federalismo como tendencia partidaria.

Por último, 1853 es el tercer momento que, al menos para algunos, constituye el verdadero origen de nuestro sistema federal. Así lo manifiestan quienes abogan por una reforma constitucional que adopte el régimen unitario y enmiende los errores cometidos por las generaciones pasadas. Inspirado en las ideas defendidas por su padrino de tesis, Carlos Melo (quien en 1897 se había doctorado con un trabajo titulado "La sugestión

\footnotetext{
${ }^{24}$ Rodríguez Larreta, C. (1902). Origen del federalismo argentino. Anales de la Facultad de Derecho y Ciencias Sociales, 1, pp. 394.
} 
universal: su manifestación en el federalismo argentino"), R. Candioti (1898) asegura que los argentinos tenemos propensiones unitarias en la sangre misma y que la decisión de los constituyentes fue determinada por la sugestión y la imitación. "llusos" (p.18), "utopistas de buena fe" (p. 25), cedieron a las presiones de un pequeño grupo de gobernadores y desoyeron "la opinión del país", la cual siempre que ha podido expresarse con libertad "ha proclamado el principio de unidad como régimen de gobierno" (p. 89). Expresiones similares se encuentran en los trabajos de Ricardo Crespo (1907, p. 33) y Teodoro Granel (1914, p. 40). Justamente lo contrario es afirmado por C. M. Vico (1905) y Martín Acevedo (1913), quienes aseveran que, a lo largo del siglo XIX, el instinto del pueblo argentino se mostró superior a la sabiduría de sus hombres eminentes, o las notabilidades, que eran mayormente unitarias. La prueba que ofrecen como justificación de su lectura es el rechazo que han generado las intervenciones provinciales: "Si realmente la masa o la mayoría quisiese la subordinación, el unitarismo, la intervención militar sería inoportuna, porque nadie iría a someter a quien pensara igual" 25 .

Al margen de esta desigual apreciación respecto de la verdadera voluntad del "pueblo argentino" en esta materia, lo cierto es que la mayoría de los tesistas se detiene a consignar y -algunos- a comentar el listado de antecedentes federales y unitarios que Juan Bautista Alberdi había presentado en sus Bases y que inspiraron parte de las discusiones de aquellos hombres eminentes de 1853. Únicamente J.A. Leiva (1905) afirma abiertamente que en el país nunca han existido antecedentes unitarios y que los señalados por Alberdi "son simplemente antecedentes nacionales que de ninguna manera importaron la existencia de una tendencia centralizadora en nuestro país" (p. 38). Más matizada es la opinión de H. Silgueira (1894), quien concibe que el unitarismo fue tan sólo un elemento "atemporizador y secundario" (p.38), en tanto que no tuvo, entre nosotros, mayores proyecciones étnicas y careció de un terreno propicio en el cual arraigar.

En la vereda opuesta, para R. Candioti (1898), U. Padilla (1900), R. Crespo (1907) y E. Marenco Aberastain (1905) los antecedentes federales apuntados por el autor de las Bases son "forzosas creaciones de [su] imaginación" (Candioti, 1898, p. 47). Siguen aquí a Juan Angel Martínez

${ }^{25}$ Vico, C. M. (1905). Una tesis unitaria. Revista Jurídica y de Ciencias Sociales, XXII, (1), p. 200. 
(1891), para quien Alberdi había inventado, "con argumentos triviales", tendencias diversificadoras de la nación que no existían (p. 16-20) ${ }^{26}$. En rigor de verdad- insisten- durante la etapa colonial lo que primó fue la uniformidad en el régimen y la represión de tendencias divergentes. No hubo comunas libres, sí, unidad en el sistema económico, en el ambiente jurídico, en los sentimientos y costumbres generales (Candioti, 1898, p.73, 138; Padilla, 1900, pp. 80-1). E. Marenco Aberastain (1905) resalta, a su vez, que "las clases ilustradas del país" buscaron establecer la unidad de régimen en los reglamentos y constituciones promulgados tras el período revolucionario y que para ello tuvieron en cuenta los antecedentes del virreinato y el hecho de que la nación estaba compuesta por el conjunto de sus habitantes y no por entidades autonómicas separadas entre sí (p. 35) 27. Como ya han advertido José Carlos Chiaramonte y Pablo Buchbinder (1992), como telón de fondo persisten las discusiones en torno a la relación de precedencia entre la nación y las provincias y las opiniones de los tesistas que se expiden sobre este punto se alinean en partes iguales detrás de Estrada y Ramos Mejía ${ }^{28}$. Por otra parte, consideran "un error contrario a la prudencia" (Padilla, 1900, p. 15) el haber buscado precedentes institucionales en las épocas anárquicas del período patrio. Comparan tal proceder con el de quien busca los antecedentes de un hombre en los instantes borrascosos de su vida. Finalmente, tanto R. Candioti como Abel Brunel recuerdan una de las leyes que, según Spencer, regula la vida de los organismos. Aquella que indica que el tránsito metódico de la evolución va de la unidad a la variedad, de la simplicidad a la complejidad y de la homogeneidad a la heterogeneidad. Sostiene que los constituyentes de

\footnotetext{
${ }^{26}$ Montes de Oca (1910) rechaza de plano la idea de que el federalismo argentino respondiera al capricho de los autores de la constitución argentina y añade como antecedente federal (a los señalados por Alberdi) la existencia de distintas corrientes colonizadoras en nuestro territorio. Amadeo (1900) discute este último elemento porque considera que estas diversas corrientes pobladoras no implantaron diferentes sistemas de colonización, ni trajeron costumbres, instituciones 0 elementos etnográficos opuestos entre sí.

${ }^{27}$ Vico escribe su texto en respuesta precisamente a la tesis de Marenco (1905), cuestiona sus conclusiones y advierte que las leyes, estatutos y las constituciones que rigieron hasta el año 26 fueron "verdaderos fantasmas constitucionales" y que la vida nacional "fue siempre federal" (p. 198).

${ }^{28} \mathrm{~A}$ modo de ejemplo, observamos que siguen a Estrada y su tesis de la prelación histórica de la nación sobre las provincias, Alfredo Gaviña (1896), Mario Carranza (1896), Carlos Octavio Bunge (1897). Se alinean detrás de Ramos Mejía y la interpretación "contractualista”, Emlio Matienzo (1894) y Ramón Moyano (1895).
} 
1853 parecen haberla olvidado y desconocen que un pueblo embrionario como el nuestro hubiera requerido en primer lugar ser organizado bajo un sistema simple para pasar luego a uno más complejo. En su lugar, se dejaron influir por la "sugestión del caudillaje" (Candioti, 1898, p. 71; Brunel, 1905, p. 25).

Como podemos observar, las discusiones en torno al origen de nuestro federalismo que, como vimos, atrajo la atención de varios tesistas, tienen como corolario la disquisición acerca de la verdadera naturaleza del mismo. ¿Se trata de un régimen necesario o contingente?, ¿puede ser pensado como original, en el sentido de que responde a necesidades y antecedentes locales o más bien debe reconocerse en él un producto importado, esencialmente foráneo? En términos generales priman las opiniones que presentan al federalismo argentino como una solución transaccional, como el resultado de las circunstancias concretas y del medio en que se implantó y se alejan de posiciones más deterministas ${ }^{29}$. Pero algunos se encargan de subrayar que tal "solución momentánea" (Crespo, 1907, p. 33), que pudo haber resultado sensata como medio de conciliación y para evitar peligros ulteriores, bien podría y debería ser objeto de revisión a partir de entonces.

En paralelo, resulta llamativo constatar, junto a Alberto Rodríguez Larreta (hermano de Carlos), que la idea de que nuestra constitución fue el resultado de una imitación completa del modelo norteamericano (también en lo que refiere a su esquema federal), era por entonces una opinión "vulgarizada por todas pares, [que] se repite en la cátedra, en los artículos de los periódicos, en los debates parlamentarios, en las reuniones políticas populares y donde quiera que nuestras leyes constituciones se discutan 0 se aduzcan" (1895, p. 13). Son varios los tesistas que consideran importante desterrar este equívoco30. C. O. Bunge (1897), por ejemplo,

29"No somos federales por una necesidad hereditaria. (...) Para nosotros, federalismo y unitarismo son simple cuestión de hecho, de circunstancia, de medio, sin que pueda predeterminar dirección alguna en ese sentido la herencia de raza", afirma C. Melo (1897, p. 33). H. Silgueira (1894), en cambio, sostiene que el federalismo es lisa y llanamente una necesidad heredada (p. 24).

${ }^{30}$ Cfr. en la misma línea a Agustín De Vedia (1899. Estudio constitucional. Organización y funciones del Poder Ejecutivo según la constitución argentina. Revista Jurídica y de Ciencias Sociales, II, p. 96). C. O. Bunge (1897) responsabiliza directamente a Del Valle de haber difundido este error. Lo acusa de ir a buscar "el abolengo de nuestro sistema representativo federal en los antecedentes políticos de los pueblos sajones y no de los hispanos" (p. 257). El mismo interés por precisar el 
señala que tanto Estrada, como Montes de Oca y Ramos Mejía aseguran que Estados Unidos había tenido una influencia secundaria en nuestra organización política. $Y$ añade: "Hemos imitado por que estábamos preparados para imitar: la imitación es lo secundario, la preparación es lo principal" (p. 346). "Nuestro federalismo (...) proviene ante todo de nosotros mismos (...), la imitación fue tan solo una colaboración oportuna" (p. 355). J. A. Leiva (1905) agrega, por otra parte, que quienes sostienen la tesis de la imitación completa niegan, al mismo tiempo, a los caudillos la capacidad intelectual y la instrucción política necesarias para poder comprender todas las especulaciones del régimen complejo que ellos propiciaban. "Incurren, pues, en una evidente contradicción, que puede darnos la medida del valor científico de la opinión mencionada" (p. 7) ${ }^{31}$.

Un último rasgo que delinea la naturaleza de nuestro federalismo para C. O. Bunge (1897) es su carácter legítimo e indestructible. Una indestructibilidad que, no obstante, resulta en seguida matizada cuando aclara que concibe ese atributo sólo en ese momento, "pues la evolución amenaza con convertirlo con los años en sistema unitario de gobierno" (p. 365). Ello mismo nos da pie para adentrarnos, seguidamente, en la segunda dimensión sobre la que se detienen los análisis de los doctorandos.

grado de influencia ejercida por el modelo norteamericano entre los constituyentes de 1853 se encuentra presente en varias tesis dedicadas a analizar la naturaleza y extensión que nuestra constitución le confiere al Poder Ejecutivo. Al respecto, cfr. Pollitzer, 2020. Sobre el lugar retórico, histórico y jurídico que adquiere, en el ámbito local, el modelo norteamericano a partir de 1890 ver Romero y Cucchi, 2017.

${ }^{31}$ En la misma línea se expresa Carlos Rodríguez Larreta unos años después: "Ha sido propósito uniforme de los escritores unitarios encarnar en la clase ignorante de las provincias el sentimiento federal y atribuirlo a las poblaciones rurales para caracterizarlo como un producto de la barbarie. Pero hay muchas pruebas de que la organización autonómica de las provincias fue iniciada y cumplida por los hombres más ilustrados de la época. Las actas que consagran nuestra formación federal están firmadas por los apellidos más notables, de los nombres tradicionales que entonces y después han constituido la clase dirigente de las ciudades argentinas" (Rodríguez Larreta, C. 1917. El triunfo del partido federal. Anales de la Facultad de Derecho y Ciencias Sociales, III, p. 180). 


\section{Interpelados por un "unitarismo práctico"}

Inmersos en un clima de balance general, el tono predominante de los trabajos doctorales que abordan la problemática del federalismo es crítico y da por supuesto la necesidad de ofrecer tanto un diagnóstico como un remedio para un paciente que retratan debilitado y, en cierto modo, en estado agónico. En principio, parece haber consenso a la hora de denunciar la distancia que separa la letra de la constitución de los resultados obtenidos en las últimas décadas y las prácticas que aún rigen la vida política. Sea que consideren conveniente adoptar un sistema unitario o lo comprendan impracticable e indeseable, las voces suenan al unísono al cuestionar que, en rigor de verdad, "no hemos tenido ni tenemos todavía, ni república, ni representación, ni federación" (Gallo,1897, p. 326). La misma afirmación figura en la tesis de Juan Agustín González Calderón (1909, p. 288). Alfredo Gaviña (1896, p. 22) y R. Crespo (1907, p. 7) precisan que no ha habido "federalismo bien entendido" en nuestra historia. Éste, agrega $\mathrm{H}$. Silgueira (1894), fue "mal interpretado en sus aplicaciones y desvirtuado en su esencia" (p. 37). Poco más que una "quimera", "un vana teoría" (Navarro, 1897, p. 83), un "traje" bajo el que se recubre una vida unitaria (Padilla Frías, $1916, \mathrm{~s} / \mathrm{p}$ ), en lugar de un régimen federal lo que hemos tenido desde 1853, dice R. Candioti (1898), es un "unitarismo práctico", en el que aún los mismos "declamadores" del federalismo (al que dicen adherir "por el deseo de ubicarse bien adulando a las masas y a los gobiernos") terminan por revelar que "eran, son y serán siempre unitarios incorregibles en el fondo y en la práctica” (p. 96).

Las voces más críticas extreman su pesimismo. En una mirada retrospectiva, nuevamente de acuerdo, advierten Juan Ángel Martínez) que el federalismo declarado no ha dado más que fracasos 32 . "Sociológicamente (...) solo ha existido en períodos anormales de nuestra vida libre y políticamente no se ha observado nunca" (Padilla, 1900, p. 98). No logró afianzar la justicia ni consolidar la paz interior- apunta Eduardo Acevedo Díaz (1910, p. 150). "No ha germinado, ni brotado, ni arraigado, ni prosperado el localismo, las autonomías, la noción siquiera del sistema federal” (Granel,1914, p. 40). Considera que por entonces agoniza, en tanto

32Juan Ángel Martínez había publicado en 1891 un libro titulado Sistema político argentino en el que señalaba que el país soportaba una "envoltura constitucional" que no se acomodaba a su organismo y llamaba a confesar "leal y francamente" que nos habíamos equivocado al elegir el régimen federal (1891, p. 7).

RHAA v.56 n.1, 2021. ISSN: 0556-5960, ISSNe 2314-1549. CC BY-NC-SA 4.0 
que ni constituye el espíritu dominante en la democracia argentina, ni es un sentimiento arraigado en el ánimo de nuestro pueblo (Padilla, 1900, p. 92). Tanto R. Candioti como O. Amadeo refieren a la carta enviada por Manuel Pizarro a Estanislao Zeballos en la que, "aquel viejo e irreconocible paladín de las autonomías provinciales" 33 afirmaba que el federalismo estaba "muerto y enterrado tal vez para siempre entre nosotros" 34 . De este modo, en vistas al futuro, la constitución es presentada por algunos como un obstáculo que limita el desenvolvimiento general y el progreso económico de nuestra sociedad (Candioti, 1898, p.138; Avellaneda, 1902, p. 128; Marenco Aberastain, 1905, p. 17).

¿En qué se basan estos diagnósticos tan oscuros y qué propuestas alientan los doctorandos? En primer lugar, advierten que los antecedentes federativos señalados por Alberdi o bien han desaparecido o bien persisten de manera muy débil. ¿Qué resta de los rasgos diferenciales de las provincias, se pregunta O. Amadeo (1900), cuando la actividad comercial manda a todas partes las mismas cosas, el ferrocarril acerca a los pueblos y los dirigentes del interior han sido educados casi todos en Buenos Aires.? La inmigración extranjera contribuye por su parte a debilitar aún más los sentimientos autonómicos. "¿Qué tradición puede mantenerse, se interroga, cuando la raza va cambiando sin cesar? (p. 35). R. Candioti (1898) también encuentra que el "espíritu diversificador" (p.128) ha huido casi por completo, en tanto que las distancias se han acortado producto del incremento de la población, el rápido crecimiento de las vías férreas, los caminos o el telégrafo.

En otro orden, el crecimiento de la población, como se sabe, no fue igual en todas partes y aún permanecían varias zonas prácticamente despobladas. A ello se le sumaba el estado todavía defectuoso de la ilustración de nuestro

${ }^{33}$ Zeballos, E. (1898). Nota a pie de página $n^{\circ} 1$. En M. Pizarro (1898). Vae victis. Carta sobre la muerte de la federación argentina. Revista de Derecho, Historia y Letras, I, (2), p. 224.

${ }^{34}$ Pizarro, M. (1898). Vae victis. Carta sobre la muerte de la federación argentina. Revista de Derecho, Historia y Letras, I (2), p. 228. Rivarola también se hace eco de la carta de Pizarro $(1904$, p. 52) y repara también en el cambio de posición manifestado por Montes de Oca. Aquél que en sus Lecciones de derecho constitucional había defendido el federalismo, una vez adquirida la experiencia de gobierno termina por aceptar que "el federalismo argentino es imposible y que la única organización conveniente para nuestro país es la del régimen de unidad de gobierno" (1908, p. 220). 
pueblo (Acevedo Díaz, 1910, p. 130). Para A. Gaviña (1896), ambos elementos conspiran contra la posibilidad de ejercer un adecuado gobierno representativo. Si gente incapaz es elegida para ocupar los cargos públicos se dará lugar a una "democracia obstructiva" y si, en cambio, los electores se restringen "a un pequeño grupo de prepotentes, a una oligarquía" (p. 48). También R. Candioti (1898) apunta contra el "nepotismo u oligarquía" (p.103) a la que daba origen el régimen vigente en el ámbito provincial. Por su parte, O. Amadeo (1900, p. 19) desafía a que alguien pruebe que las provincias están mejor gobernadas que los territorios federales en razón de su autonomía. La respuesta no se hizo esperar, ya que ese mismo año Vico escribe en las páginas de la Revista Jurídica y de Ciencias Sociales que los territorios nacionales son "la parte peor gobernada de la nación", donde las necesidades locales no necesariamente son atendidas, ni sus funcionarios son controlados o responsables y donde los conflictos con las autoridades son más frecuentes. "Es un desconsolador espejo esa administraciónconcluye-, mírense en él los que desean para la República un régimen de centralización administrativa"35.

Si los elementos que decidieron la adopción del sistema federal para la Argentina en 1853 han perdido influencia 0 se han debilitado profundamente, los elementos centralistas aún subsisten y algunos se han robustecido en los últimos tiempos de una manera "asombrosa e inesperada" (Amadeo 1900, p. 30). Es más, según E. Marenco Aberastain (1905), éstos son ahora más poderosos porque "están reforzados por el amor a la patria común, cuyos vínculos no eran tan fuertes en la época revolucionaria" (p. 46). Entre esos elementos el que más se destaca es el crecimiento del centralismo porteño tras la federalización de la ciudad de Buenos Aires y el consiguiente aumento del desequilibrio entre ésta y el resto de las provincias. Varios tesistas dan cuenta del aflujo migratorio de las "clases ricas" y de "los elementos intelectuales" del interior hacia Buenos Aires. La mitad de los estudiantes de la Universidad de Buenos Airescomentan $\mathrm{O}$. Amadeo y R. Candioti- son provincianos y en su mayoría se quedan definitivamente allí. En efecto, de los 53 tesistas seleccionados para este trabajo, todos hombres, 28 son de Buenos Aires o de la Capital Federal y 17 provienen del resto de las provincias ${ }^{36}$. En el discurso pronunciado por

\footnotetext{
${ }^{35}$ Vico, C. M. (1905). Una tesis unitaria. Revista Jurídica y de Ciencias Sociales, XXII, (1), p. 202.

${ }^{36}$ Faltan datos sobre 8 tesistas. Si tomamos una muestra mayor, que incluya a todos los estudiantes que optaron por realizar su tesis doctoral sobre un tema vinculado al
} 
Ernesto Padilla en el acto de colación de grados de 1896, este graduado de origen tucumano que más adelante se desempeñaría como profesor suplente de filosofía general y tendría una importante trayectoria política, critica con dureza el achatamiento de la vida en las provincias, el abandono de la lucha intelectual y activa que allí se observa y que atribuye:

al egoísmo de sus hombres que prefieren para teatro de sus esfuerzos esta ciudad, aquí donde los esfuerzos sobran, dando lugar a que el raquitismo, la anemia vayan enfermando las instituciones con la esterilidad a que condenan el suelo que está destinado a verlas crecer $^{37}$.

¿Qué puede hacer esta juventud para que su acción resulte realmente eficaz?, se pregunta Ramón Alsina (1909) en otro de estos discursos.

La juventud universitaria debe ir a las provincias, donde faltan hombres [responde]. Debe tratar de que su esfuerzo no se pierda estérilmente donde no se lo necesita, y conservarlo para levantar el nivel económico, moral e intelectual de las provincias donde su acción ha de ser siempre saludable y fecunda ${ }^{38}$.

El centralismo porteño se manifiesta también en el ámbito de la opinión pública. R. Candioti (1898) explica que aquello que gusta o conviene propagar a los periodistas porteños (o mejor dicho, a sus tres grandes diarios) conforma, al día siguiente, la opinión de la capital y, dos días más tarde, la opinión de la prensa provinciana. De modo que lo que se señala como "la manifestación de la conciencia nacional" no es más que el reflejo de la prensa porteña (p. 131). Claramente, concluye, en Argentina el desarrollo de sus partes componentes no está en proporción con la diferenciación que la Constitución exige. En paralelo, J. C. Cruz (1896) menciona la influencia "demasiado sugestiva" que la opinión pública de la capital ejerce sobre el Congreso. Observa que, por este motivo, las leyes son aplaudidas o desprestigiadas antes de entrar en vigor en todo el país.

derecho constitucional entre 1890 y 1916 (347), la relación entre los alumnos que provienen de Buenos Aires y aquellos que son oriundos del interior se empareja ( $37 \%$ y $36 \%$ respectivamente, aunque faltan datos para un $27 \%$ ).

${ }^{37}$ Padilla, E. (1896). Discurso de colación de grados. Anales de la Universidad, XI, p. 148.

${ }^{38}$ Alsina, R. (1909). Discurso colación de grados. Revista Jurídica y de Ciencias Sociales, XXVI, (2), p.106. 
De todos modos, reflexiona que como la opinión pública de la Capital Federal es ilustrada, las consecuencias de su prejuicio no son, en el fondo, perjudiciales, y que "si se contraría en algo el sistema federal, se tiende a realizar el ideal de Jefferson: la república patricia, en que el poder es ejercido siempre por los mejores, por esa aristocracia de selección que según algunos autores es la verdadera democracia" (p. 101).

La creciente acción administrativa de la nación sobre las provincias también es subrayada por los doctorandos. Como botón de prueba, señalan que aquéllas están llenas de empleados y de obras nacionales. El gobierno nacional, insiste $\mathrm{O}$. Amadeo unos años después de graduarse, es el primer empresario de obras públicas, es el educador casi exclusivo de la niñez y la juventud y el principal promotor de la industria ${ }^{39}$.

Bajo el punto de vista económico y fiscal el cuadro dista de ser más optimista. Las provincias son presentadas como estados débiles, insolventes en su mayoría, que no cuentan con los recursos necesarios para sostener la maquinaria administrativa y mucho menos para fomentar su industria, la agricultura, la ganadería o el comercio. Para hacer frente a sus necesidades o bien se ven forzadas a subir los impuestos o a contraer empréstitos que luego no pueden pagar. El estado nacional asume así la responsabilidad ante los acreedores externos y termina por subvencionar a las provincias con el tesoro nacional. Algo "insólito" en la historia de las federaciones, según apunta R. Crespo (1907, p. 37), en consonancia con E. Prack (1893, p. 211), R. Candioti (1898, p. 123), U. Padilla (1900, pp. 100-2) y E. Marenco Aberastain (1905, p. 58). Quienes destacan esta anomalía, pero mantienen su compromiso con la causa federal, proponen, como medidas correctivas, la reunión de varios estados provinciales en estados poderosos capaces de hacer realidad la autonomía de estas jurisdicciones, la reducción de los sueldos y dietas legislativas, la supresión de puestos públicos innecesarios y la revisión de los impuestos internos.

Finalmente, el "unitarismo práctico" en que se vive se ha llevado puesta la tan elogiada y teóricamente defendida autonomía municipal. Según R. Candioti (1898), los mismos que apoyan el federalismo no comprenden la autonomía comunal y no quieren reconocer otra que la provincial, vale decir, "una mayor, por medio de la cual puedan los caudillos y sus círculos colocarse frente al gobierno de la nación" (p.127). La realidad es que los

${ }^{39}$ Amadeo, O. (1908). El neo-unitarismo. La Nación. 26-10-1908, p. 5. 
poderes que se encuentran sobre la comuna no hacen más que trabar su organización, inmiscuirse en sus asuntos internos, impedir el ejercicio amplio de su libertad electoral y adueñarse del manejo de sus rentas ${ }^{40}$.

Frente a semejante panorama, algunas voces (aunque minoritarias) reclaman por una revisión sustancial de la constitución vigente. U. Padilla, por ejemplo, fundamenta su posición con un argumento que considera casi evidente:

Si es cierto que los estados se organizan siempre sobre la base de sus instituciones anteriores, la República Argentina ha debido adoptar el unitarismo, que es el principio consagrado por sus antecedentes más apreciables y numerosos. Si, por el contrario, y es lo que parece más exacto, la forma que adopta un pueblo para su gobierno está mas bien determinada por las circunstancias que rodean su organización, las que presidieron el establecimiento de nuestra carta fundamental han variado sustancialmente, y se impone, por tanto, un cambio en el sistema gubernativo, y la consagración de un régimen más acorde con las conveniencias actuales de la nación y más conforme con las prácticas de nuestra democracia (Padilla, 1900, pp. 78-9).

R. Candioti (1898) también sostiene que la reforma constitucional es necesaria y la califica como "un imperativo categórico, penetrado de fuerte y enérgica precisión, al cual no podemos desobedecer impunemente" ( $p$. 154). Para T. Granel (1914), ella obedece a una necesidad "real, visible y palpable" (p. 96). Por su parte, O. Amadeo (1900) entiende que se trata de una "evolución orgánica y fatal", acorde a la marcha general del mundo moderno (p. 39). En ello acuerda con C. O. Bunge (1897, pp. 364-5) y J. A. Leiva (1905, p. 42) quienes- amén de su defensa del federalismo- no dejan de constatar como una tendencia "universal" el avance de la centralización política. Francia, Alemania, Austria, Estados Unidos son los ejemplos invocados por todos ellos.

Aluden a una reforma "completamente factible" (Padilla, 1900, p. 96), que aspire en primer lugar, a trasparentar y legalizar las prácticas vigentes. Como había argumentado Manuel Pizarro (a quien suele citar este grupo de tesistas) o reiterará luego R. Rivarola $(1904,1908)$, “una constitución

\footnotetext{
${ }^{40}$ Entre 1891 y 1916 se defendieron 23 tesis que abordaron la cuestión del gobierno municipal. Tal vez una de las descripciones más críticas y descarnadas sobre la vida municipal se encuentra en el trabajo de Juan José Díaz Arana (1902).
} 
unitaria permitirá armonizar los hechos con el derecho y suprimirá todo lo discrecional y arbitrario de las prácticas actuales" (Pizarro, 1898, p. 230). Lo que se busca es, entonces, que el gobierno nacional tenga el mismo poder que en la actualidad, pero asuma la responsabilidad de la que hoy se ve eximido.

Así, proponen establecer una única legislatura para todo el territorio de la nación. Las razones esgrimidas son de índole política y económica. Por un lado, anhelan que de este modo puedan corregirse- al menos en parte- las anomalías presentes en la representación política, puesto que los poderes de provincia "no siempre interpretan la voluntad popular, ni se inspiran en los sagrados intereses de la colectividad" (Padilla, 1900, p. 102). Por otro, conceptúan las legislaturas provinciales de "inútiles y perjudiciales" (Candioti, 1898, p.165), y a sus ministerios como "aparatosos y caros" (Amadeo, 1908, p. 5). Resulta imperativo disminuir los empleos y las funciones públicas y combatir así la tan denunciada "empleomanía", mal que según C. O. Brunel (1905) se sostiene en la pretensión por parte de un número elevado de individuos de ocupar puestos públicos rentados en lugar de contribuir con su actividad a la industria, el comercio u otras ramas del trabajo "productivo" (p. 27). E. Acevedo Díaz (1910) suscribe: "El federalismo es caro: cada provincia costea dos gobiernos, el propio y el nacional" (p. 157), aunque reconoce haber escuchado la opinión contraria en la cátedra de Finanzas de la Facultad (por parte de su profesor suplente). En un artículo publicado en la Revista Argentina de Ciencia Política, Isidoro Ruiz Moreno (1911) ${ }^{41}$ confirma que esta opinión no se discute y constituye uno de los argumentos de mayor fuerza levantados por quienes se oponen al actual régimen federal. El considera, sin embargo, que ésta no puede ser la razón de primer orden, sencillamente porque es falsa. Intenta demostrar en su texto que, de hecho, el régimen unitario sería incluso más caro que el federal.

\footnotetext{
${ }^{41}$ Ruiz Moreno, I. (1911). Federalismo y unitarismo ante los gastos públicos. Revista Argentina de Ciencias Políticas, II, pp. 153-166. Había cursado sus primeros años en esta casa de estudios, pero se doctoró en la Universidad de Córdoba en 1898. En dicha universidad, fue profesor de Finanzas y Sociología; también dictó clases de Finanzas y de Historia del Derecho Argentino en la Universidad Nacional de la Plata. Luego de su paso por el Congreso (08-12). Como diputado, fue incorporado a la cátedra de Derecho Internacional Público de la Facultad de Derecho de la Universidad de Buenos Aires, y a partir de 1919 sería nombrado titular.
} 
Otra propuesta discutida por los tesistas refiere a la posibilidad de unificar también el poder judicial en toda la nación ${ }^{42}$. Además del ahorro económico que tal modificación ocasionaría, algunos piensan que, al librar a los jueces de la sujeción de los círculos provinciales, su independencia quedaría mejor resguardada (Candioti, 1898, p. 112; Acevedo Díaz, 1910, p. 150; Granel, 1914, p. 171). Esta apreciación es rebatida por Nicolás González Yramain (1910), quien además de advertir que tal modificación (aunque compatible con el sistema republicano) implicaría necesariamente la adopción del sistema unitario- con el que no acuerda-43, señala que los actuales funcionarios que administran justicia en el ámbito nacional son bien remunerados, tienen asegurada su inmovilidad y aun así "da vergüenza referir sus corruptelas" y constatar cómo "ceden a las mismas influencias que perturban y corrompen la magistratura de las provincias" (p. 79). La misma opinión es compartida por Emilio Moyano (1911, p. 85).

Por último, señalan la conveniencia de unificar la legislación electoral para todo el país y la necesidad de reemplazar los partidos locales por grandes partidos nacionales. Como explica R. Candioti (1898), éstos se diferenciarían entre sí respecto de la posición que cada uno adopte sobre cuestiones de interés general, como por ejemplo, la tensión entre el libre comercio y el proteccionismo, la rivalidad económica entre el litoral y el interior o la separación de la Iglesia y el Estado.

Por lo general, quienes apoyan estas reformas y consienten en que puedan ser introducidas de forma paulatina, preservan el federalismo como un "objeto de aspiración para el futuro" (Padilla, 1900, p. 137) con la esperanza de restaurarlo "cuando ya no nos pueda ser tan gravoso" (Avellaneda, 1902, p. 129), cuando "seamos dignos y estemos preparados" (Candioti, 1898, p. 151). En cambio, la mayoría de los tesistas, aún cuando cuestionen ciertas prácticas o señalen imperfecciones en la carta constitucional, se mantienen firmes defensores del esquema federal. "No puedo concebir ni la más remota idea de que alguna vez pueda modificarse la forma actual de

\footnotetext{
${ }^{42}$ Entre 1909 y 1912, cinco estudiantes eligieron puntualmente como tema para su disertación doctoral: "Organización de la justicia en la República: si conviene o no su unificación”. Ellos fueron Eduardo Graña, Nicolás González Yramain, Mario Casas, Emilio Moyano y Félix Valle.

${ }^{43}$ E. Graña (1909), M. Casas (1911) y F. Valle (1911) también rechazan la adopción del unitarismo, pero consideran que la unificación de los fueros a nivel nacional (con la que sí acuerdan) no afectaría al régimen federal.
} 
gobierno por la unitaria", sentencia O. Navarro (1897, p. 84). "No es posible en esta república otro sistema de gobierno", confirma C.O. Bunge (1897, p. 2), haciéndose eco de lo que, a su entender, constituye la opinión de la mayoría. Es que el defecto no anida en el régimen, sino que el mal está en su práctica viciosa (Alvarez Prado, 1906, p. 209). Los pedidos, entonces, apuntan a que se respete la letra constitucional, que la nación procure dar a las provincias vida económica propia, que no viole sus autonomías con "fútiles pretextos", o que "imparta el ejemplo desde su alto puesto el primer magistrado de la República, mostrándose respetuoso de los fueros de los otros dos poderes" (González Yramain, 1910, p. 80).

Dos cuestiones más agitan los debates vinculados a la realidad del sistema federal argentino en torno al cambio de siglo. La primera tiene que ver con la capital nacional. Entre 1896 y 1907 seis doctorandos eligieron directamente esta temática para sus disertaciones finales. En su trabajo, J. C. Cruz (1896) ofrece una revisión histórica de las discusiones que tuvieron lugar sobre la ubicación de la capital y, aunque estima que como solución teórica hubiera sido preferible establecerla en otro sitio (en alguna meseta de la provincia de Córdoba), considera que en ese momento su traslado resultaría inconveniente (p. 61). Se encuentra en clara minoría, junto a Anibal Noceti (1898), porque el resto de los tesistas se pronuncian en sentido contrario (Mercado, 1896; De la Vega, 1898; Alvarez Prado, 1906; Sarmiento Laspiur, 190744). Luis Alvarez Prado (1906), por caso, encuentra la capitalización de Buenos Aires como la gran responsable de los males de la política argentina y propone fundar una nueva ciudad, de tamaño reducido donde deberán vivir los funcionarios por el tiempo de su mandato y los legisladores durante el período parlamentario. Buenos Aires no será así, "la dominadora tiránica en la economía, la política y el crédito" (p. 256). Sugiere que se conforme una comisión de personas idóneas (entre las cuales debe haber médicos, abogados, ingenieros y políticos, entre otros) para decidir la nueva localización. No cree conveniente, por otra parte, hacer que la ciudad de Bs.As. sea devuelta a la provincia, salvo que ésta fuera dividida en dos o tres partes.

La segunda cuestión tiene que ver con las intervenciones provinciales. Como en el caso de la capitalización de Buenos Aires, además de encontrar comentarios puntuales sobre éstas por parte del resto de los doctorandos,

44También se pronuncian a favor del traslado de la capital los estudiantes Alvarez (1894), Padilla (1900) y Acevedo (1913).

RHAA v.56 n.1, 2021. ISSN: 0556-5960, ISSNe 2314-1549. CC BY-NC-SA 4.0 
entre 1893 y 1909 fueron nueve las tesis dedicadas exclusivamente a su estudio. Es difícil encontrar un precepto más discutido, opina Pedro Elicagaray (1896), ha dado lugar a intensos debates en el parlamento y "ha subido a la cátedra con todo el calor que inspira el estudio de los principios fundamentales de nuestro régimen político" (Elicagaray, 1896, p.15). Entre los tesistas suscita una apreciación desigual. Valgan, como ejemplo, las siguientes afirmaciones:

Cuando se oye pronunciar la palabra intervención al gobierno federal, los pueblos se inquietan, se alarman, porque saben que los espera una gran calamidad y no el fallo levantado e imparcial del poder central (Navarro, 1897, p. 81).

Las intervenciones son recibidas por las provincias con aplausos. (...) Hay más sonrisas en los rostros y en las empresas más vigor, los delegados del poder central son despedidos al terminar su misión con verdadero pesar de los pueblos intervenidos (Amadeo, 1900, p. 35).

Mientras algunos cargan las tintas sobre los abusos cometidos y encuentran que ellas "sólo han servido para humillar a los pueblos, hollar las autonomías provinciales y burlar la opinión pública" (Navarro, 1897, p. 81), otros recuerdan que, "honradamente practicada, [la intervención] es la mejor garantía de las instituciones de un pueblo libre" (Elicagaray, 1896, p. 77). Exigen, eso sí, una regla uniforme de aplicabilidad, una reglamentación de los preceptos constitucionales en la que se precise, por ejemplo, cuál es el poder que debe decretar la intervención, a quién le compete pronunciarse acerca de su finalización y cuáles son las facultades de que ha de gozar el interventor (Galdeano, 1893, p. 13; Aguirre, 1907, p. 26; Acevedo, 1913, p. 149). En orden a desincentivar las intervenciones, E. Acevedo Díaz (1910, p. 135) sugiere que diputados y legisladores sea elegidos simultáneamente al presidente de la República y duren en sus mandatos tanto tiempo como aquél. Agrega también, que el gobernador saliente no pueda ser elegido legislador nacional hasta pasado un período de dos años. $Y$ frente a la posible objeción que alguno pudiera esgrimir, al sostener que de este modo se privaría al gobierno de consejeros con experiencia, responde que, en la historia parlamentaria argentina, los ex-gobernadores han tenido una participación pasiva en las deliberaciones del Senado. 


\section{Consideraciones finales}

La exploración de este conjunto de textos nos acerca de un modo privilegiado al interior de la Facultad de Derecho y a los análisis y discusiones que en ella tuvieron lugar en torno al cambio de siglo respecto del federalismo argentino. Si bien algunas de estas voces expresaron sus opiniones también desde otros ámbitos, como la prensa, el Parlamento y a través de publicaciones que alcanzaron mayor repercusión, aquí hemos buscado reponer sus primeras intervenciones-en algunos casos- y reconstruir el diálogo entre docentes y alumnos gestado en este particular espacio de sociabilidad.

Hemos visto que las autoridades de la Facultad incentivaron el estudio de algunos preceptos constitucionales relativos, por ejemplo, al deslinde de las facultades nacionales y provinciales, y difundieron desde la Revista de la Universidad o los Anales de la Facultad las opiniones de sus docentes o graduados respecto de la conveniencia 0 no de instaurar un régimen unitario. Por su parte, cerca de un $15 \%$ de los alumnos que optaron por un tema de derecho constitucional para escribir sus disertaciones doctorales entre 1890 y 1916, lo hizo para referirse a la problemática del federalismo. Como es esperable, en esta polifonía de voces encontramos una serie de acuerdos y otros puntos de disenso. Entre los primeros, destacamos la escasa atención concedida a una discusión doctrinal que confrontara teóricamente las bondades del federalismo 0 el unitarismo, respectivamente. Tampoco detectamos una perspectiva de análisis comparado (a excepción del caso norteamericano), en el que la constitución y las prácticas locales sean cotejadas con otras experiencias regionales 0 europeas. La mirada está puesta, como expresamos, exclusivamente en el espacio local y se bifurca, en forma equitativa, hacia el pasado y hacia la coyuntura en la cual se produjeron esos escritos. La experiencia histórica y los antecedentes jurídicos de la Constitución de 1853 son visitados para justificar, impugnar o discutir la vigencia del esquema federal adoptado por aquélla. Amén del peso relativo que cada uno adjudica a los distintos elementos que dieron origen a nuestro federalismo o de las razones que, para cada quien, explican la decisión de aquellos constituyentes en esta materia, la opinión mayoritaria destaca como notas características de nuestro régimen su carácter transaccional y originario. 
También es compartido el tono crítico utilizado para delinear los contornos de una realidad en la que la conjunción de determinadas decisiones (como la capitalización de Buenos Aires), los cambios producidos por el aluvión inmigratorio, el desigual crecimiento económico y poblacional y el alejamiento por parte de ciertas prácticas políticas de la letra y los preceptos constitucionales se plasmaba en un federalismo extremadamente centralizado45. Como observa Darío Roldán (2015) para el caso de la Revista Argentina de Ciencias Políticas, notamos que, al menos en estas tesis, el acento no está puesto en cuestionar la legitimidad de un régimen por la ausencia de las garantías necesarias para una participación electoral trasparente, sino en señalar la ineficacia o el falseamiento de ciertas instituciones y prácticas políticas para representar adecuadamente a la sociedad, para limitar la acción del poder y para permitir el desenvolvimiento general y el progreso económico de la sociedad en su conjunto.

Los disensos más significativos afloran entre los tesistas que se refieren explícitamente a la coyuntura y a la necesidad o inconveniencia de instaurar un régimen unitario. Cada grupo se presenta como el portavoz de una opinión mayoritaria (Bunge, 1897, p. 2; Candioti, 1898, p. 156) y aunque en teoría ambos admiren el sistema federal, hay quienes entienden que éste tan solo podrá encarnarse con posterioridad. Mientras tanto, estiman que "en la consolidación de la República sobre una base unitaria está la solución de dificultades graves, económicas y sociológicas que tienen, a nuestro modo de ver, íntima correlación con el régimen político" (Padilla, 1900, pp. 102-3).

Finalmente, constatamos que gran parte de los argumentos y las propuestas que R. Rivarola presenta en sus obras de 1904 y 1908 ya se encuentran anticipadas por varios tesistas, quienes recogen, a su vez, las enseñanzas de Juan Angel Martínez entre otros. No hemos encontrado, de todos modos, en ninguna de estas tesis revisadas la sugerencia (presente en el unitarismo alentado por Rivarola) de modificar el Senado Nacional para hacer de él un ámbito de representación de la diversidad de intereses sociales. Las discusiones en torno a la naturaleza del sufragio o la conveniencia de adoptar un régimen parlamentario, también centrales en la

\footnotetext{
${ }^{45}$ Estas lecturas han sido objeto de revisión por parte de la historiografía de los últimos años. Como botón de muestra, Paula Alonso (2015) desafía la imagen aquí esbozada sobre el peso abrumador que habría tenido el estado nacional y el presidente sobre las provincias afines del siglo XIX.
} 
propuesta de Rivarola, tampoco aparecen en los textos trabajados para este artículo pero sí fueron objeto de debate en otras disertaciones doctorales del mismo período (Pollitzer 2018, 2020). Las opiniones de J.N.Matienzo son invocadas con menor frecuencia, y por lo general lo hacen los tesistas que circunscriben sus análisis a las reformas de la constitución introducidas en 1860 .

\section{Fuentes}

Acevedo Díaz, E. (1910). El unipersonalismo político argentino. En: E. Acevedo Díaz. Los Nuestros (pp. 121-162). M. García.

Aguirre, C. (1907). Intervenciones. Imprenta José Tragant.

Alvarez, Heráclito. (1894) Juicio Político. Imprenta y papelería "El Americano".

Alvarez Prado, L. (1906). La capital de la Nación. Imprenta y encuadernación Nereo Doblas.

Amadeo, O. (1900). Derecho constitucional. Evolución unitaria. Imprenta El día.

Avellaneda, C. (1902). El sistema federal y la tradición histórica argentina. Tesis de doctorado inédita. Facultad de Derecho y Ciencias Sociales, Universidad de Buenos Aires.

Brunel, A. (1905). La república unitaria: antecedentes. Tesis de doctorado inédita. Facultad de Derecho y Ciencias Sociales. Universidad de Buenos Aires.

Bunge, C. O. (1897). El federalismo argentino. M. Biedma.

Candioti, M. (1920). Bibliografía doctoral de la Universidad de Buenos Aires y catálogo cronológico de las tesis en su primer centenario (18211920). Ministerio de Agricultura.

Candioti, R. (1898). Tendencias unificadoras de la Argentina. La Unión.

Carranza, M. (1896). Atribuciones nacionales y provinciales. Imprenta Juan A. Alsina. 
Casas, M. (1911). Organización de la justicia. Tesis de doctorado inédita. Facultad de Derecho y Ciencias Sociales, Universidad de Buenos Aires.

Crespo, R. (1907). Unidad de régimen. Imprenta de J.A. Berra.

Cruz, J. C. (1896). La Capital. Imprenta de J.A. Berra.

De la Vega, J. M. (1898). La capital federal y las instituciones federativas. Compañía Sud-Americana Billetes de Banco.

Díaz Arana, J. J. (1902). El régimen municipal argentino. Imprenta y casa editorial Coni Hermanos.

Elicagaray, P. (1896). Intervención federal. Compañía Sud-Americana Billetes de Banco.

Galdeano, P. (1893). Intervención federal: comentario a los artículos $5^{\circ}$ y $6^{\circ}$ de la Constitución Nacional. Argos.

Gallo, V. (1897). Juicio político. Impr. Pablo E. Coni e hijos.

Gaviña, A. (1896). Sistema de gobierno de la República argentina. Pech.

González Calderón, J. A. (1909). El poder legislativo en los estatutos, reglamentos y constituciones de la nación y las provincias. Valerio Abeledo editor.

González Yramain, N. (1910). Organización de la justicia; si conviene o no su unificación. Tesis de doctorado inédita. Faculta de Derecho y Ciencias Sociales, Universidad de Buenos Aires.

Granel, T. (1914). Reformas a la Constitución en 1860. Compañía SudAmericana Billetes de Banco.

Graña, E. (1909). Organización de la justicia. Tesis de doctorado inédita. Facultad de Derecho y Ciencias Sociales, Universidad de Buenos Aires.

Leiva, J. A. (1905). Origen del federalismo argentino. Tesis de doctorado inédita. Facultad de Derecho y Ciencias Sociales, Universidad de Buenos Aires.

Marenco Aberastain, E. (1905). Tendencias unitarias en la República Argentina. Las Ciencias.

Martínez, J. A. (1891). Sistema político argentino. Soler Hermanos. 
Matienzo, E. (1894). ¿Cuál es el alcance de la intervención? Imprenta Europea.

Melo, C. F. (1897). La sugestión universal: su manifestación en el federalismo argentino. Imprenta J.A. Berra.

Mercado, A. (1896). Cuestión Capital Federal. Imprenta de J.A. Berra.

Moyano, R. (1895). Federalismo argentino. Roma editorial.

Moyano, E. (1911). Organización de la justicia. Si conviene o no su unificación. Tesis de doctorado inédita. Facultad de Derecho y Ciencias Sociales, Universidad de Buenos Aires.

Montes de Oca, M. A. (1910). Lecciones de derecho constitucional. Imprenta y litografía La Buenos Aires.

Naón, R. (1896). Deslindes de las facultades nacionales y provinciales. A. Monkes.

Noceti, A. (1898). La capital. Compañía Sud-Americana Billetes de Banco.

Navarro, Octavio (1897). Autonomías provinciales. Imprenta J.A. Berra.

Padilla, U. F. (1900). El unitarismo argentino. Guillermo Kraft.

Padilla Frías, M. (1916). Poderes gubernamentales conservados por las provincias. Tesis de doctorado inédita. Faculta de Derecho y Ciencias Sociales, Universidad de Buenos Aires.

Rivarola, R. (1904). Partidos políticos. Unitario y Federal. F. Lajouane editor.

Rivarola, R. (1908). Del régimen federativo al unitario. Peuser.

Rodríguez Larreta, A. (1895). La nación y las provincias. Imprenta J.A. Berra.

Sarmiento Laspiur, E. (1907). La Capital. Adolfo Grau.

Silgueira, J. H. (1894). Federalismo. El correo español.

Valle, F. (1911). Organización de la justicia. Tesis de doctorado inédita. Faculta de Derecho y Ciencias Sociales, Universidad de Buenos Aires. 


\section{Bibliografía}

Alonso, P. (2006). Reflexiones y testimonios en torno de la Reforma electoral, 1910-1916. En: D. Roldán (Comp.) Crear la democracia. La Revista Argentina de Ciencias Políticas y el debate en torno a la República verdadera (pp. 187-236). Fondo de Cultura Económica.

Alonso, P. (2015). Dinámicas federales en las dos últimas décadas del siglo XIX. P. Alonso y B. Bragoni (Eds.). El sistema federal argentino. Debates y coyunturas (1860-1910). (pp. 199-221). Edhasa.

Chiaramonte, J.C. y Buchbinder, P. (1992). Provincias, caudillos, nación y la historiografía constitucional argentina. Anuario del IEHS, (VII), pp. 93120. https://dialnet.unirioja.es/servlet/articulo?codigo $=5162023$

Leiva, A. D. (1989). Los primeros ochenta años de historiografía jurídica argentina. Lecciones y Ensayos, (53), pp. 75-87. http://www.derecho.uba.ar/publicaciones/lye/revistas/53/los-primerosochenta-anos-de-la-historiografia-juridica-argentina.pdf

Pollitzer, M. (2020). Discusiones sobre el gobierno parlamentario en la Facultad de Derecho 1890-1920. Revista de Historia del Derecho, (59), pp. 1-36.

http://www.scielo.org.ar/scielo.php?script=sci_arttext\&pid=S1853$17842020000100001 \&$ lng=es\&nrm=iso\&tlng=es

Pollitzer, M. (2018). Abstencionismo político y sufragio obligatorio a comienzos del siglo XX: la voz de la Facultad de Derecho y Ciencias Sociales de la Universidad de Buenos Aires. Polhis, 21, (11), p. 122160. http://www.polhis.com.ar/index.php/polhis/article/view/291

Roldán, D. (2015). El debate sobre el federalismo y las opacidades de la política argentina en el Centenario. En: P. Alonso y B. Bragoni (Eds). El sistema federal argentino. Debates y coyunturas (1860-1910), pp. 223-249. Edhasa.

Romero, A. L. y Cucchi, L. (2017). El «modelo» norteamericano en la reglamentación de las intervenciones federales en la Argentina decimonónica. Debates en el Congreso Nacional (1869 y 1894). Anuario de Estudios Americanos, 74, (2), pp. 615-642. https://doi.org/10.3989/aeamer.2017.v74.i2 
Tanzi, J. (2011). La enseñanza del derecho constitucional en la Facultad de Derecho de la Universidad de Buenos Aires. Academia. Revista sobre la enseñanza del derecho, 9, (17), pp. 85-112.

Tau Anzoátegui, V. (1996). El derecho en la vida finisecular de J. A. García. Revista de Historia del Derecho, (24), pp. 293-404. 